\title{
RELACCÕES ENTRE A PERSONALIDADE DOS PACIENTES E A SOBREVIVÊNCIA APÓS O TRANSPLANTE DE MEDULA ÓSSEA: REVISÃO DA LITERATURA
}

\author{
Rodrigo Sanches Peres \\ Manoel Antônio dos Santos"
}

\begin{abstract}
RESUMO. O transplante de medula óssea (TMO) é um procedimento médico complexo, utilizado no tratamento de diversas enfermidades graves quando as terapêuticas convencionais não oferecem um bom prognóstico. Pesquisas recentes sugerem que a personalidade do sujeito submetido ao referido procedimento é um dos fatores psicossociais mais determinantes para sua sobrevivência. O presente estudo visa a avaliar de forma sistemática os artigos científicos dedicados à pesquisa das relações entre a personalidade dos pacientes e a sobrevivência pós-TMO. A coleta de dados foi realizada mediante a execução de buscas eletrônicas em diferentes sistemas de identificação bibliográfica especializados disponíveis on-line. Os resultados obtidos evidenciam a necessidade de novas pesquisas, pois a efetiva compreensão das relações entre a personalidade e a sobrevivência de pacientes submetidos ao TMO pode colaborar na prevenção e manutenção do bem-estar biopsicossocial desses sujeitos.
\end{abstract}

Palavras-chave: personalidade, transplante de medula óssea, sobrevivência.

\section{LINKINGS BETWEEN PATIENTS' PERSONALITY AND BONE MARROW TRANSPLANT SURVIVAL: BIBLIOGRAPHICAL REVIEW}

\begin{abstract}
Bone marrow transplant (BMT) is a medical procedure used in case of serious illness treatment, that is, when the conventional therapeutic modalities do not offer a good prognostic. Recent researches suggest that, the patients' personality is a decisive psychosocial factor for adaptation and recovery after BMT procedure. The present paper intends to evaluate the scientific productions regarding the patients' personality and its relation to post-BMT survival. Data collection was carried out by using on-line searches in the different bibliographical identification systems available. The results obtained suggest the need for new researches, because understanding the relation between the patients' personality and the post-BMT survival can aid the individual/patient in both, in the prevention, as well as in the maintenance of the bio-psychosocial well being.
\end{abstract}

Key words: Personality, bone marrow transplant, survival.

\section{RELACIONES ENTRE LA PERSONALIDAD DE LOS PACIENTES Y LA SUPERVIVENCIA DESPUÉS DEL TRASPLANTE DE MÉDULA ÓSEA: REVISIÓN DE LA LITERATURA}

\begin{abstract}
RESUMEN. El trasplante de médula ósea (TMO) es un procedimiento médico complejo, utilizado en el tratamiento de diversas enfermedades graves cuando las terapias convencionales no ofrecen un buen pronóstico. Investigaciones recientes sugieren que la personalidad del sujeto sometido a dicho procedimiento es uno de los factores psicosociales más determinantes para su supervivencia. El presente estudio pretende evaluar sistemáticamente los artículos científicos dedicados a la investigación de las relaciones entre la personalidad de los pacientes y la supervivencia pos TMO. La recogida de datos fue realizada mediante la ejecución de búsquedas electrónicas en diferentes sistemas de identificación bibliográfica especializados on-line. Los resultados obtenidos dejan en evidencia la necesidad de nuevas investigaciones, pues la efectiva comprensión de las relaciones entre la personalidad y la supervivencia de pacientes sometidos al TMO puede colaborar en la prevención y mantenimiento del bienestar biopsicosocial de esos sujetos.

Palabras-clave: personalidad, trasplante de médula ósea, supervivencia.
\end{abstract}

* Psicólogo. Doutorando em Psicologia pela Universidade de São Paulo (Faculdade de Filosofia, Ciências e Letras de Ribeirão Preto).

\# Psicólogo. Doutor em Psicologia Clínica, professor do Departamento de Psicologia e Educação da Universidade de São Paulo (Faculdade de Filosofia, Ciências e Letras de Ribeirão Preto), bolsista de Produtividade em Pesquisa. 


\section{ASPECTOS BIOMÉDICOS DO TRANSPLANTE DE MEDULA ÓSSEA}

O transplante de medula óssea (TMO) é um procedimento médico complexo, utilizado freqüentemente nas duas últimas décadas no tratamento de uma série de enfermidades graves quando as terapêuticas convencionais não oferecem um bom prognóstico (Thomas, 2000). O método em questão envolve a infusão de suspensões de células-tronco e visa basicamente a reverter a aplasia medular decorrente de doenças hematológicas, oncológicas e onco-hematológicas. Assim, oferece possibilidade de recuperação a pacientes portadores de tumores sólidos, leucemias, anemias, linfomas, hemoglobinopatias e outras enfermidades potencialmente letais, de modo que pode ser considerado um dos maiores avanços da medicina moderna (Job, 1994).

Não obstante, cumpre assinalar que o TMO não se afigura como um método plenamente resolutivo. Tratase, na realidade, de um procedimento agressivo, que pode tanto recuperar a vida do paciente quanto conduzi-lo ao óbito. Esse paradoxo ocorre basicamente porque a imunossupressão induzida pelo regime de condicionamento pré-TMO torna o receptor temporariamente vulnerável a complicações que acarretam riscos não apenas à sua integridade física, mas também à sua própria vida. Em função disso, cerca de $40 \%$ dos pacientes submetidos a essa terapêutica apresentam evolução clínica fatal (Tabak, 2000). Conseqüentemente, o TMO muitas vezes é visto como um procedimento ambivalente, ao mesmo tempo salvador e ameaçador (Lesko, 1989; Contel \& cols., 2000).

\section{ASPECTOS PSICOSSOCIAIS DO TRANSPLANTE DE MEDULA ÓSSEA}

Até os anos 1980, as investigações científicas relacionadas ao TMO focalizavam apenas seus aspectos biomédicos. Tais investigações contribuíram para o aprimoramento dos procedimentos técnicos da modalidade terapêutica em questão, de maneira que o TMO deixou de ser visto como uma estratégia experimental e passou a ser considerado um método convencional para o tratamento de diversas enfermidades graves. Atualmente, contudo, nota-se que houve uma significativa mudança no foco das pesquisas, pois há um número cada vez maior de estudos dedicados aos aspectos psicossociais do TMO (Neitzert \& cols., 1998).

Recentemente o exame das relações entre certas variáveis psicossociais e a sobrevivência de pacientes submetidos à terapêutica em questão vem se destacando como um tema de pesquisa de grande relevância ${ }^{1}$. Em linhas gerais, os estudos voltados ao referido tema indicam que o apoio de amigos e familiares, a tendência à ansiedade e à depressão, os recursos adaptativos prévios e as estratégias de enfrentamento adotadas pelo paciente perante a doença e o tratamento são alguns dos mais importantes preditores psicossociais da reabilitação pós-TMO (Andrykowski, Bruchl, Brady. \& Henslee-Downey, 1995, Colon \& cols., 1994, Contel \& cols., 2000, Jenkins, Linington \& Whitakker, 1991, Wingard, 1994).

Além disso, diversas pesquisas sugerem que a personalidade - ou seja, a unidade individual resultante da interação de fatores biológicos, sociais e culturais que determina o comportamento (D'Andrea, 2000; Fadiman \& Frager, 1979, Lazarus \& Monat, 1984, Ramos Jr., 1991) - do paciente submetido ao TMO é um fator relacionado à sua sobrevivência (Andrykowski, 1994, Andrykowski, Brady \& HensleeDowney, 1994, Fromm, Andrykowski \& Hunt, 1995, Lesko, 1989, Wettergren, Langius, Björkholm, \& Björvell, 1997, Wolcott, Wellisch, Fawzy \& Landsverk, 1986). Não obstante, muitas dessas pesquisas não são dedicadas especificamente ao delineamento da interface entre as duas variáveis em questão.

\section{OBJETIVO}

O presente estudo tem como objetivo avaliar de forma sistemática os artigos científicos voltados ao exame das relações entre a personalidade dos pacientes e a sobrevivência pós-TMO.

\section{JUSTIFICATIVA}

O conhecimento resultante do presente estudo pode subsidiar o delineamento de um panorama global da produção científica concernente ao tema em

\footnotetext{
Vale ressaltar que a influência de variáveis psicossociais no curso de diversos tratamentos médicos tem sido objeto de uma série de estudos publicados nos últimos anos. Em um desses estudos, Pereira, Rosa \& Haddad (2002) relacionaram a adaptação psicológica à sobrevivência de indivíduos submetidos ao transplante cardíaco. Pressman $\&$ cols. (2005), por outro lado, identificaram ligações entre a extensão e a qualidade da rede de suporte social e a resposta imunológica a vacinas. Já Silva Filho \& Souza (2004) se dedicaram à investigação de determinantes emocionais na evolução clínica de pacientes soropositivos.
} 
questão e, desse modo, fornecer elementos: a) para o aprimoramento das práticas assistenciais desenvolvidas no âmbito multidisciplinar junto a pacientes submetidos ao TMO e b) para a realização de estudos posteriores, voltados mais especificamente aos aspectos psicossociais do TMO.

\section{MÉTODO}

\section{Estratégia de recuperação bibliográfica: procedimentos} para coleta do material

O material necessário para o levantamento bibliográfico foi coletado mediante a execução de buscas eletrônicas em diferentes bases de dados online. Optou-se por utilizar tal procedimento levando-se em consideração que atualmente os sistemas de identificação bibliográfica disponíveis na World Wide Web se destacam como recursos de divulgação científica extremamente valiosos. Porém, todas as bases de dados existentes apresentam limitações específicas. Assim sendo, com o intuito de possibilitar o desenvolvimento de uma revisão completa da literatura, foram consultados cinco sistemas de identificação bibliográfica distintos, sendo quatro deles internacionais e um nacional, a saber: MedLine, PsycINFO, Web of Science, LILACS e Index Psi.

As buscas limitaram-se ao cruzamento dos verbetes "personalidade" (personality) e "transplante de medula óssea" (bone marrow transplantation). Considerou-se pertinente a utilização de tais termos, que são particularmente abrangentes, tendo-se em vista que, em consultas preliminares às referidas bases de dados, o emprego de descritores mais específicos restringiu demasiadamente o número de referências localizadas. Vale destacar também que os sistemas de identificação bibliográfica consultados foram configurados para localizar as referências que apresentavam os descritores em questão em todos os campos de busca possíveis. Esse procedimento foi adotado com o objetivo de possibilitar a localização de um número de referências maior do que aquele que se poderia coligir mediante a especificação dos campos a serem pesquisados.

Os artigos localizados foram recuperados na íntegra, com vista a proporcionar uma análise mais apurada do material. Dessa forma, as fontes secundárias de informação - os sistemas on-line de identificação bibliográfica - foram empregadas apenas como meio a partir do qual se pode ter acesso às fontes primárias - os artigos publicados em periódicos indexados. A busca das fontes, tanto primárias quanto secundárias, foi feita inicialmente junto ao serviço de periódicos da Biblioteca Central do Campus de Ribeirão Preto da Universidade de São Paulo (USP). Posteriormente, a busca de fontes primárias foi complementada mediante a solicitação de periódicos junto a outras bibliotecas - nacionais e internacionais - vinculadas ao Programa de Comutação Bibliográfica (COMUT).

\section{Análise inicial do material: procedimentos para seleção das referências}

O título e a autoria de todas as referências obtidas a partir da busca eletrônica foram inicialmente checados com o intuito de subsidiar a eliminação de eventuais repetições. A seguir, os resumos dos artigos restantes foram submetidos a uma leitura preliminar e seletiva, que teve como propósito determinar o material efetivamente pertinente ao presente estudo. Essa leitura foi norteada por alguns critérios de inclusão e exclusão, como será exposto a seguir.

\section{Critérios de inclusão}

Foram considerados pertinentes os artigos: a) publicados em periódicos indexados; b) escritos em língua inglesa, francesa, espanhola ou portuguesa; c) publicados entre 1981 e 2001; d) que apresentam resultados empíricos ou oriundos de revisão da literatura; e) que possuem como objeto principal de pesquisa a personalidade de pacientes adultos com indicação ou submetidos ao TMO e f) que se dedicam aos aspectos psicossociais do TMO e apresentam, direta ou indiretamente, contribuições ao tema.

\section{Critérios de exclusão}

Além de formular os referidos critérios de inclusão, optou-se por estabelecer também alguns critérios de exclusão, com o intuito de tornar a seleção dos estudos mais precisa. Assim sendo, foram descartados: a) capítulos de livros, dissertações ou teses; b) artigos que se referem estritamente aos aspectos médicos do TMO; c) artigos que focalizam os resultados de programas de atendimento médico ou psicológico a pacientes com indicação ou submetidos ao TMO; d) artigos que analisam essencialmente os resultados de programas de atendimento médico ou psicológico a familiares de pacientes submetidos ao TMO; e) artigos que enfatizam o exame das repercussões do TMO no doador ou na equipe médica e f) artigos que visam à validação de instrumentos. 
Análise avançada do material: procedimentos para apreciação da qualidade metodológica das referências selecionadas

A qualidade metodológica do material selecionado foi apreciada mediante a leitura analítica e integral de cada um dos trabalhos. Para melhor organização e compreensão, os resultados oriundos dessa leitura foram tabulados a partir da identificação de 10 dimensões de análise, a saber: 1) modalidade de produção científica; 2) instituição e país de origem; 3) temas estudados; 4) principais objetivos; 5) número de sujeitos; 6) estratégia metodológica; 7) tipo de delineamento de pesquisa; 8) referencial teórico; 9) instrumentos utilizados e 10) principais resultados obtidos.

\section{RESULTADOS: APRESENTAÇÃO E DISCUSSÃO}

A partir do cruzamento dos verbetes selecionados foram obtidas 51 referências. Dentre os sistemas eletrônicos de identificação bibliográfica empregados, o MedLine foi o que se mostrou mais profícuo, visto que possibilitou a localização de 28 artigos. O LILACS e o Index $P s i$, por outro lado, não indicaram sequer uma referência relacionada ao tema pesquisado, o que evidencia de antemão a ausência de estudos dedicados ao exame das relações entre a personalidade dos pacientes e a sobrevivência pós-TMO em publicações latino-americanas indexadas. Ademais, tal discrepância sugere que os aspectos biomédicos do TMO ainda são um tema de pesquisa recorrente, pois o MedLine privilegia notadamente revistas médicas.

A seleção preliminar do material obtido apontou que 10 das 51 referências foram indicadas mais de uma vez pelas diferentes bases de dados empregadas, totalizando 13 repetições. Conseqüentemente, o número de artigos reduziu-se de 51 para 38 , pois as repetições foram automaticamente desconsideradas. A seguir, as referências inicialmente selecionadas foram avaliadas em função dos critérios de inclusão e exclusão estabelecidos. Tal procedimento eliminou mais um considerável número de artigos, pois 20 deles não atendiam aos critérios adotados. Em função disso, foram efetivamente consideradas pertinentes ao presente estudo apenas 18 das 51 referências inicialmente obtidas.

A notável diferença verificada entre o número de referências obtidas mediante a busca nos sistemas eletrônicos de identificação bibliográfica empregados (51) e a quantidade de artigos considerados efetivamente pertinentes ao presente estudo (18) já era, de certa forma, esperada, em virtude do estabelecimento de critérios de inclusão e exclusão rigorosos. Cumpre assinalar, contudo, que tais critérios se mostraram de extrema importância, pois permitiram selecionar adequadamente as referências inicialmente obtidas, conferindo, conseqüentemente, maior precisão ao presente estudo.

A avaliação da modalidade de produção científica do material selecionado indica que a maioria absoluta dos artigos apresenta dados empíricos oriundos de trabalhos originais. Em virtude do número reduzido de pesquisas dedicadas especificamente ao exame das relações entre a personalidade dos pacientes e a sobrevivência pós-TMO, pode-se propor que esse objeto de estudo ainda não foi devidamente explorado. Nesse sentido, a prevalência de pesquisas empíricas pode ser considerada positiva, uma vez que a realização de trabalhos com esse caráter indubitavelmente contribui para uma melhor compreensão do objeto de estudo em questão. Por outro lado, a inexistência de trabalhos de revisão bibliográfica pode ser considerada desfavorável, pois se sabe que tais trabalhos possibilitam o delineamento de um panorama abrangente da produção científica relativa a um determinado tema e fornecem valiosas indicações para novas pesquisas, de modo que possuem um inegável valor prático (Alves-Mazzotti \& Gewandsnadjer, 2000).

Verificou-se com a segunda dimensão de análise adotada que 35 dos 47 pesquisadores responsáveis pela redação dos artigos selecionados são filiados a universidades norte-americanas. A John Hopkins University, em Baltimore (Maryland), foi a instituição que mais se destacou nesse sentido, com 9 pesquisadores (Baker, Marcellus, Zabora, Polland \& Jodrey, 1997, Curbow, Somerfield, Baker, Wingard \& Legro,1993, Somerfield, Curbow, Wingard, Baker \& Fogart, 1996). Na realidade, o fato de a grande maioria dos estudos disponíveis na literatura científica acerca do tema em questão ter sido conduzida em instituições norte-americanas já era previsto, uma vez que, segundo dados da International Bone Marrow Transplantation Registry $^{2}$, os Estados Unidos da América possuem mais centros especializados em TMO do que qualquer outro país do mundo.

No que se refere aos temas pesquisados, os trabalhos de Curbow e cols. (1993), Jenkins e cols. (1991), Fife e cols. (2000), Litwins, Rodrigue e Weiner (1994), Molassiotis, Van den Akker e Bougton (1997a), Mundy e cols. (2000), Somerfield e cols. (1996), Soussain e Amiel-Lebigre (1992)

2 Serviço norte-americano que concentra dados estatísticos dos principais centros de TMO do mundo. 
priorizam basicamente a investigação das repercussões psicossociais do TMO. Bressi, Capra, Brambilla e Deliliers (1995), Baker e cols. (1997) e Rodrigue, Boggs, Weiner e Behen (1993), por outro lado, focalizam a avaliação do funcionamento psicológico de candidatos ao TMO, ao passo que Weitzner, A.; Lehninger, Sullivan e Fields (1999) apresentam um estudo de caso de uma paciente borderline com indicação para o referido procedimento. Já os artigos de Andrykowski e cols (1994), Molassiotis, Van den Akker, Milligan e Goldman (1997b), Pearman (1998) e Sullivan, Szkrumelak e Hoffman (1999a; 1999b) privilegiam especificamente a investigação das variáveis psicossociais associadas ao TMO. O trabalho de Neuser (1988), por fim, tem como principal objeto a influência das características de personalidade na sobrevivência dos pacientes submetidos a essa terapêutica.

Nota-se, destarte, que, ao contrário do que se poderia esperar, a maior parte dos autores dos artigos selecionados focaliza - em detrimento da avaliação da personalidade de pacientes com indicação ou submetidos ao TMO - a investigação do funcionamento psicossocial dos sujeitos. Porém, a leitura integral dos artigos indica que os autores efetivamente consideram a personalidade dos pacientes um dos mais relevantes fatores psicossociais envolvidos no TMO. Desse modo, poder-se-ia questionar por que então eles não se dedicam especificamente ao estudo da personalidade dos pacientes. Em linhas gerais, é possível supor que os referidos autores optaram pelo exame de variáveis mais específicas - e mais passíveis de mensuração basicamente porque a avaliação da personalidade envolve uma série de dificuldades metodológicas decorrentes da abrangência do constructo em questão.

Os artigos que priorizam basicamente o exame das repercussões psicossociais do TMO apresentam objetivos distintos, a saber: a) a investigação das estratégias de enfrentamento utilizadas pelos sujeitos para lidar com as sequielas do tratamento (Somerfield \& cols., 1996); b) a avaliação da qualidade de vida (Litwins \& cols., 1994), da morbidade psicossocial (Jenkins \& cols., 1991), dos níveis de estresse (Fife \& cols., 2000, Mundy \& cols., 2000), ansiedade e depressão dos pacientes (Soussain \& Amiel-Lebigre, 1992); c) a análise do suporte social e familiar recebido (Molassiotis \& cols., 1997a) e d) o delineamento das mudanças pessoais desencadeadas pela terapêutica em questão (Curbow \& cols., 1993).

Em contrapartida, os artigos dedicados especificamente às variáveis psicossociais associadas à sobrevivência pós-TMO investigam, em linhas gerais, o valor preditivo das estratégias de enfrentamento (Molassiotis \& cols., 1997b) e das respostas adaptativas dos pacientes (Sullivan \& cols., 1999a; 1999b), do suporte familiar e social recebido (Pearman, 1998), das condições psicológicas e da qualidade de vida (Andrykowski \& cols., 1994) na evolução do tratamento. Neuser (1988), por sua vez, pesquisou as relações entre a personalidade de pacientes e a sobrevivência pós-TMO, ao passo que Weitzner e cols. (1999) apresentam considerações éticas a partir de um estudo de caso de uma paciente borderline com indicação para o referido procedimento. Por fim, o funcionamento psicológico, as respostas adaptativas e os níveis de distresse de candidatos a essa terapêutica foram investigados, respectivamente, por Rodrigue e cols. (1993), Bressi e cols. (1995) e Baker e cols. (1997).

Dos dezoito artigos selecionados, nove utilizaram um número de sujeitos inferior a cinqüenta (Andrykowski \& cols., 1994, Bressi \& cols., 1995, Jenkins \& cols., 1991, Molassiotis \& cols., 1997b, Mundy e cols., 2000, Neuser, 1988, Pearman, 1998, Soussain \& Amiel-Lebigre, 1992, Weitzner \& cols., 1999), três empregaram de cinquienta a cem pacientes (Litwins \& cols., 1994, Molassiotis \& cols., 1997a, Rodrigue \& cols., 1993) e seis tiveram como participantes mais de cem indivíduos (Baker \& cols., 1997, Curbow \& cols., 1993, Fife \& cols., 2000, Somerfield \& cols., 1996, Sullivan \& cols., 1999a, 1999b). Obviamente, o fato de a maior parte dos estudos selecionados empregar um número relativamente reduzido de sujeitos é desfavorável, pois compromete a elaboração de hipóteses generalizáveis. No entanto, trata-se de uma limitação compreensível, visto que, em virtude da complexidade do procedimento, poucos centros de TMO são capazes de atender a mais de cem pacientes por ano. Dessa forma, a realização de estudos multicêntricos pode ser considerada a única forma exequível de contornar tal dificuldade.

A análise da estratégia metodológica da qual os autores lançaram mão indica que as abordagens quantitativas, empregadas em treze estudos, foram notadamente privilegiadas em detrimento das abordagens qualitativas, utilizadas em apenas um artigo (Weitzner \& cols., 1999), e das abordagens combinadas (quantiqualitativas), adotadas em quatro pesquisas (Molassiotis, 1997a, Soussain \& AmielLebigre, 1992, Sullivan \& cols., 1999a, 1999b). Possivelmente, a predileção pelas abordagens quantitativas se encontra intimamente associada ao fato de a maior parte dos autores ser filiada a instituições norte-americanas, uma vez que os Estados 
Unidos da América possuem uma notória tradição neste tipo de enfoque metodológico de pesquisa.

Não obstante, é preciso mencionar que a quantificação de dados, a despeito de ser um procedimento merecedor de crédito, não raro é erroneamente valorizada de modo desmesurado. Isso ocorre porque muitos pesquisadores não levam em consideração que as análises qualitativas permitem, sem deixar a desejar em termos de cientificidade, a superação do paradigma positivista, que conduz à naturalização dos fenômenos psicológicos. De qualquer forma, vale destacar que, assim como apontam Valles (1997) e Pereira (1999), confrontar as abordagens quantitativa e qualitativa com o intuito de identificar uma ordem de precedência entre ambas é impertinente, uma vez que cada uma delas apresenta vantagens e limitações específicas.

A maioria dos autores dos artigos selecionados avaliou retrospectivamente os examinados (Curbow \& cols., 1993, Jenkins \& cols., 1991, Litwins \& cols., 1994, Molassiotis \& cols., 1997a, Molassiotis \& cols., 1997b, Pearman, 1998, Somerfiled e cols., 1996, Soussain \& Amiel-Lebigre, 1992, Sullivan \& cols., 1999a, 1999b) e adotou o delineamento transversal de pesquisa (Baker \& cols., 1997, Curbow \& cols., 1993, Jenkins \& cols., 1991, Litwins \& cols., 1994, Molassiotis, 1997a, Rodrigue \& cols., 1993, Somerfiled \& cols., 1996, Soussain \& Amiel-Lebigre, 1992, Sullivan \& cols., 1999a, Weitzner \& cols., 1999).

Levando-se em conta o objetivo principal de cada um dos artigos em questão, pode-se considerar que, em linhas gerais, o delineamento de pesquisa adotado pela maioria dos autores foi adequado. Cumpre assinalar, contudo, que o delineamento longitudinal seria mais compatível com os objetivos propostos por Curbow e cols. (1993), Somerfield e cols. (1995) e Molassiotis e cols (1997a), pois possibilitaria a comparação de dados obtidos em diferentes momentos de avaliação.

As teorias de orientação cognitivo-comportamental foram notadamente privilegiadas pelos autores dos artigos selecionados. As teorias de orientação psicodinâmica, por sua vez, foram empregadas em apenas três estudos (Soussain \& Amiel-Lebigre, 1992, Sullivan \& cols., 1999a, 1999b). Tal discrepância também pode estar associada ao fato de haver uma prevalência de pesquisadores filiados a instituições norte-americanas entre os autores dos artigos selecionados, levando-se em conta que as teorias de orientação cognitivo-comportamental encontram nos Estados Unidos da América um terreno propício para seu desenvolvimento e utilização.
No que concerne à nona modalidade de análise preconizada, verificou-se que em apenas quatro dos dezoito artigos selecionados foram utilizadas técnicas que focalizam especificamente a investigação da personalidade (Bressi e cols., 1995, Jenkins \& cols., 1991, Neuser, 1988, Rodrigue \& cols., 1993). Dentre tais técnicas, a mais frequiente foi o Minnessota Multiphasic Personality Inventory ${ }^{3}$ (MMPI), adotado por Rodrigue e cols. (1993) e Bressi e cols. (1995). Nos artigos restantes empregaram-se basicamente escalas e inventários que priorizam, em linhas gerais, a avaliação do suporte familiar e social, das estratégias de enfrentamento, do ajustamento social e dos níveis de ansiedade, depressão e estresse dos pacientes. Nesse sentido, o Profile of Mood States ${ }^{4}$ (POMS) se destacou como o instrumento preferido dos autores, pois foi utilizado em seis estudos (Andrykowski \& cols 1994, Baker \& cols., 1997, Curbow \& cols., 1993, Molassiotis \& cols., 1997b, Mundy \& cols., 2000, Somerfield \& cols., 1996).

Faz-se necessário destacar ainda que em três dos dezoito artigos selecionados foram utilizados roteiros semi-estruturados de entrevista (Weitzner \& cols., 1999, Sullivan \& cols., 1999a, 1999b). Assim sendo, nota-se que os autores não lançaram mão de técnicas projetivas. Em linhas gerais, esse fato pode ser justificado levando-se em conta que os instrumentos psicométricos fornecem - mais do que as técnicas de exame psicológico de outros tipos - elementos propícios para análises quantitativas e estatísticas. Conseqüentemente, são plenamente compatíveis com a abordagem metodológica adotada pelos autores.

Em que pese a isso, é possível cogitar que os instrumentos psicométricos empregados nos artigos selecionados tendem a gerar apenas subsídios parciais para a compreensão do objeto em questão, já que não são voltados à avaliação da personalidade. Ademais, é preciso salientar que os instrumentos projetivos eliciam dados mais ricos acerca do psiquismo do examinado, já que, exigindo um intenso grau de criação e elaboração pessoal, viabilizam a investigação dos processos mentais profundos

2 O MMPI é constituído por 566 questões referentes a 26 traços de personalidade. Trata-se de um dos mais conhecidos instrumentos de avaliação psicológica, uma vez que vem sendo mundialmente empregado desde 1943, quando sua primeira versão foi publicada nos Estados Unidos da América.

3 O POMS visa à identificação de transtornos de humor e é composto de 65 questões, que se dividem em 6 subescalas. A primeira versão do instrumento foi publicada em 1971, nos Estados Unidos da América, e sua utilização tem se tornado cada vez mais difundida, notadamente na última década. 
(Güntert, 2000). Portanto, a despeito dos problemas metodológicos inerentes à abordagem qualitativa, intuitiva e clínica que preconizam, não deveriam ser totalmente preteridos pelos autores.

Os resultados das pesquisas de Rodrigue e cols. (1993), Bressi e cols. (1995) e Baker e cols. (1997) demonstram que uma parcela significativa de pacientes, a despeito de adotar estratégias de enfrentamento adaptativas e não apresentar indícios psicopatológicos significativos no que concerne ao funcionamento da personalidade, possuía sintomas de distresse no período pré-TMO. Tais dados são congruentes com os assinalados por Jenkins e cols. (1991), Soussain e Amiel-Lebigre (1992), Mundy e cols. (2000) e Fife e cols. (2000), que apontam que a maioria dos sujeitos submetidos à terapêutica em questão apresenta escores mais elevados de ansiedade, depressão e estresse no período de condicionamento do que na fase de recuperação após o tratamento. Os autores sugerem que, possivelmente, isso ocorre porque antes do TMO os pacientes temem as complicações que podem ser desencadeadas pelo referido procedimento e depois se sentem satisfeitos por terem conseguido sobreviver.

Além disso, de acordo com Litwins e cols. (1994) e Somerfield e cols. (1996), após o TMO os sujeitos tendem a optar por estratégias de enfrentamento adaptativas para lidar com as repercussões do tratamento, a ter um bom reajustamento psicossocial e a apresentar níveis de qualidade de vida semelhantes ao de indivíduos submetidos a modalidades terapêuticas mais convencionais e menos ameaçadoras, tais como a quimioterapia. Cumpre assinalar, por fim, que, sob a ótica da maior parte dos pacientes avaliados, o suporte social e familiar recebido foi adequado e o tratamento trouxe mais mudanças positivas (principalmente do ponto de vista dos relacionamentos interpessoais) do que negativas (Curbow \& cols., 1993, Molassiotis \& cols., 1997a). Tais resultados, entretanto, não são compatíveis com os achados de outros estudos, que apontam que o TMO repercute de maneira negativa na qualidade de vida dos indivíduos submetidos a esse procedimento (Ferrell \& cols., 1992, Molassiotis \& Morris, 1999).

Os artigos que focalizam especificamente as relações entre as variáveis psicossociais e a recuperação pós-TMO concluem que pacientes desesperançosos, que não buscam ajuda para lidar com as limitações impostas pela doença, apresentam um funcionamento psicológico imaturo, obtêm escores elevados de ansiedade, estresse e depressão, possuem uma qualidade de vida comprometida antes do tratamento, adotam estratégias de enfrentamento pouco adaptativas e recebem suporte social e familiar inadequado (Andrykowski \& cols, 1994, Molassiotis \& cols., 1997b, Neuser, 1988, Pearman, 1998, Sullivan \& cols., 1999a, 1999b), usualmente encontram acentuadas dificuldades de ajustamento psicossocial, correm maiores riscos de serem acometidos pela reincidência da enfermidade ou por outras complicações e, conseqüentemente, alcançam uma sobrevivência reduzida.

\section{CONSIDERAÇÕES FINAIS}

De modo geral, os autores dos estudos selecionados realizaram pesquisas de delineamento retrospectivo e transversal, adotaram abordagens quantitativas e visaram basicamente: a) à investigação das variáveis psicossociais associadas à sobrevivência pós-TMO; b) ao estudo das repercussões psicossociais do procedimento em questão e c) à avaliação das condições psicológicas de candidatos à referida terapêutica. Além disso, apoiaram-se em teorias de orientação cognitivo-comportamental, utilizaram grupos relativamente reduzidos de participantes e lançaram mão de instrumentos psicométricos que examinam mais especificamente o suporte familiar e social recebido, as estratégias de enfrentamento e os níveis de ansiedade, depressão, estresse e ajustamento psicossocial dos pacientes. Assim sendo, os artigos selecionados fornecem, em sua maioria, contribuições essencialmente parciais para a compreensão das relações entre a personalidade e a sobrevivência de sujeitos submetidos ao TMO.

Destarte o presente estudo indica que a personalidade dos pacientes pode ser considerada, atualmente, a variável psicossocial associada ao TMO a respeito da qual se tem menos conhecimento, o que evidencia a necessidade de novas pesquisas dedicadas especificamente a esse assunto. Entretanto, ressalte-se que, possivelmente, os pesquisadores interessados em uma melhor compreensão desse objeto de estudo obterão resultados mais profícuos se adotarem desenhos metodológicos inovadores em relação aos que foram privilegiados pelos autores dos artigos selecionados. Nesse sentido, o emprego de abordagens qualitativas, a utilização de teorias de orientação psicodinâmica e a aplicação de instrumentos - sejam eles psicométricos, projetivos ou de qualquer outro tipo - voltados especificamente à avaliação da personalidade parecem as alternativas mais indicadas ${ }^{5}$.

5 Duas dissertações de mestrado (Almeida, 1998; TorranoMasetti, 2000) não publicadas apresentam algumas das 


\section{REFERÊNCIAS}

Almeida, A. C. (1998). Avaliação psicológica e qualidade de vida de pacientes submetidos ao transplante de medula óssea. Dissertação de Mestrado Não-Publicada, Programa de Pós-Graduação em Psicologia, Universidade de São Paulo, Ribeirão Preto.

Alves-Mazzotti, A. J. \& Gewandsnadjer, F. (2000). O método nas ciências naturais e sociais: pesquisa quantitativa $e$ qualitativa. São Paulo: Pioneira.

Andrykowski, M. A. (1994). Psychosocial factors in bone marrow transplantation: A review and recommendations for research. Bone Marrow Transplantation, 13, 357-375.

Andrykowski, M. A., Brady, M. J. \& Henslee-Downey, P. J. (1994). Psychosocial factors in predictive of survival after allogeneic bone marrow transplantation. Psychosomatic Medicine, 56(5), 432-439.

Andrykowski, M. A., Bruchl, S., Brady. M. J. \& HensleeDowney, P. J. (1995). Physical and psychosocial status of adults one-year after bone marrow transplantation: A prospective study. Bone Marrow Transplantation, 15, 837845.

Baker, F., Marcellus, D., Zabora, J., Polland, A. \& Jodrey, D. (1997). Psychological distress among adult patients being evaluated for bone marrow transplantation. Psychosomatics, 38(1), 10-19.

Bressi, C., Capra, E., Brambilla, V. \& Deliliers, G. (1995). Psychological variables and adjustment to illness in subjects undergoing bone marrow transplantation. New Trends in Experimental and Clinical Psychiatry, 11(1), 25-37.

Colon, E. A, Callies, A. L., Popkin, M. K. \& Mc Glave, P. B. (1994). Depressed mood and others variables related to boné marrow transplant survival in acute leukemia. Psychosomatics, 32, 420-425.

Contel, J. O. B., Sponholz Jr., A., Torrano-Masetti, L. M., Almeida, A. C., Oliveira, E. A., Jesus, J. S., Santos, M. A., Loureiro, S. R. \& Voltarelli, J. C. (2000). Aspectos psicológicos e psiquiátricos do transplante de medula óssea. Medicina, 33, 294-231.

Curbow, B., Somerfield, M. R., Baker, F., Wingard, J. R. \& Legro, M. W. (1993). Personal changes, dispositional optimism and psychological adjustment to bone marrow transplantation. Journal of Behavioral Medicine, 16(5), 423-443.

referidas características e foram desenvolvidas no Brasil junto à Unidade de Transplante de Medula Óssea do Hospital das Clínicas da Faculdade de Medicina de Ribeirão Preto da Universidade de São Paulo (HCFMRPUSP). Tais dissertações indicam que o exame das relações entre a personalidade dos pacientes e a sobrevivência pósTMO é um objeto de estudo consideravelmente promissor e que a exploração de novas estratégias metodológicas se destaca como o melhor caminho para tornar possível sua efetiva compreensão.
D'Andrea, F. F. (2000). Desenvolvimento da personalidade: enfoque psicodinâmico. Rio de Janeiro: Bertrandt.

Fadiman, J. \& Frager, R. (1979). Teorias da personalidade (C. P. Sampaio \& S. Safolié, Trads.). São Paulo: Harper \& Row.

Ferrell, B., Schmidt, G. M., Rhiner, M., Whitehead, C., Fonbuena, P. \& Forman, S. J. (1992). The meaning of quality of life for bone marrow transplantation survivors. Part 1: The impact of bone marrow transplant on quality of life. Cancer Nursing, 15(3), 153-160.

Fife, B. L., Huster, G. A., Cornetta, K. G., Kennedy, V. N., Akard, L. P. \& Broun, E. R. (2000). Longitudinal study of adaptation to the stress of bone marrow transplantation. Journal of Clinical Oncology, 18(7), 1539-1549.

Fromm, K., Andrykowski, M. A. \& Hunt, J. (1995). Positive and negative psychosocial sequelae of bone marrow transplantation: Implications for quality of life assessment. Journal of Behavioral Medicine, 19(3), 221-233.

Güntert, A. E. V. A. (2000). Técnicas projetivas: o geral e o singular em avaliação psicológica. Em F. F. Sisto, E. T. B Sbardelini \& R. Primi (Orgs.), Contextos e questões da avaliação psicológica. (pp.77-84). São Paulo: Casa do Psicólogo.

Jenkins, P. L., Linigton, A. \& Whitakker, J. A. (1991). A retrospective study of psychosocial morbidity in bone marrow recipients. Psychosomatics, 32(1), 65-71.

Job, F. (1994). Transplante de medula óssea. Revista do Hospital das Clínicas de Porto Alegre, 14(31), 5-11.

Lazarus, R. S. \& Monat, A. (1984). Personalidade (V. Ribeiro, Trad.). Rio de Janeiro: Zahar.

Lesko, L. M. (1989). Bone marrow transplantation. In J. C. Holland \& J. H. Rowland (Eds.), Handbook of Psychooncology. (pp.163-173). New York: Oxford University.

Litwins, N. M., Rodrigue, J. R. \& Weiner, R. S. (1994). Quality of life in adult recipients of bone marrow transplantation. Psychological Reports, 75, 323-328.

Molassiotis, A., Van den Akker, O. B. A. \& Bougton, B. J. (1997a). Perceived social suport, family environment and psychosocial recovery in bone marrow transplantation long-term survivors. Social and Scientific Medicine, 44(3), 317-325.

Molassiotis, A., Van den Akker, O. B. A., Milligan, D. W. \& Goldman, J. M. (1997b). Symptom distress, coping style and biological variables as predictors of survival after bone marrow transplantation. Journal of Psychosomatic Research, 42(3), 275-285.

Molassiotis, A. \& Morris, P. J. (1999). Quality of life in patients with chronic myeloid leukemia after unrelated bone marrow transplantation. Cancer Nursing, 22(5), 340349.

Mundy, E. A., Blanchard, E. B., Cirenza, E., Gargiulo, J., Maloy, B. \& Blanchard, B. (2000). Posttraumatic stress disorder in breast cancer patients following autologus bone marrow transplantation or conventional cancer treatments. Behavioral Research and Therapy, 38(10), 1015-1027. 
Neitzert, C. S., Ritvo, P., Dancey, J., Weiser, K., Murray, C. \& Avery, J. (1998). The psychosocial impact of bone marrow transplantation: A review of the literature. Bone Marrow Transplantation, 22, 409-422.

Neuser, J. (1988). Personality and survival time after bone marrow transplantation. Journal of Psychosomatic Research, 32(4-5), 451-455.

Pearman, T. P. (1998). Mortality and morbidity following bone marrow transplantation: Predictive utility of psychological variables. Dissertation Abstracts International, 58,(7-B), 3932.

Pereira, A. A. M., Rosa, J. T. \& Haddad, N. (2002). Adaptação psicológica, fatores de risco e probabilidade de sobrevida em transplante cardíaco. Mudanças, 10(1), 4161.

Pereira, J. C. R. (1999). Análise de dados qualitativos. São Paulo: EDUSP.

Pressman, S. D., Cohen, S., Miller, E., Barkin, A., Rabin, B. S. \& Treanor, J. J. (2005). Loneliness, social network size, and immune response to influenza vaccination in college freshmen. Health Psychology, 24(3), 297-306.

Ramos Jr., J. (1991). Personalidade. São Paulo: Sarvier.

Rodrigue, J. R., Boggs, S. R., Weiner, R. S. \& Behen, J. M. (1993). Mood, coping style and personality functioning among adult bone marrow transplant candidates. Psychosomatics, 34(2), 159-165.

Silva Filho, N. \& Souza, L. R. (2004). Variações adaptativas, relações objetais e evolução clínica em pacientes com infecção pelo HIV-1, doentes ou não. Mudanças, 12(1), 94-114.

Somerfield, M. R., Curbow, B., Wingard, J. R., Baker, F. \& Fogart, L. A. (1996). Coping with the physical and psychosocial sequelae of bone marrow transplantation among long-term survivors. Journal of Behavioral Medicine, 19(2), 163-184.

Soussain, C. \& Amiel-Lebigre, F. (1992). Troubles psychologiques rencontrés pendant la période postgreffe de moelle osseuse. Bulletin du Cancer, 79(12), 1135-1148.

Sullivan, A. K., Szkrumelak, N. \& Hoffman, L. H. (1999a). Psychological risk factors and early complications after bone marrow transplantation in adults. Bone Marrow Transplantation, 24, 1109-1120.
Sullivan, A. K., Szkrumelak, N. \& Hoffman, L. H. (1999b). Psychiatry assessment of candidates for bone marrow transplantation: A psychodynamically-oriented approach. Internacional Journal of Psychiatry and Medicine, 29(1), 13-28.

Tabak, D. G. (2000). Transplante de medula óssea na leucemia mielóide crônica. Medicina, 33, 232-240.

Thomas, E. D. (2000). Bone marrow transplantation: A historical review. Medicina, 33, 209-218.

Torrano-Masetti, L. M. (2000). Repetição do des-conhecido: contribuições da psicossomática psicanalítica na recaída do câncer hematológico. Dissertação de Mestrado NãoPublicada, Programa de Pós-Graduação em Psicologia, Universidade de São Paulo, Ribeirão Preto.

Weitzner, M. A., Lehninger, F., Sullivan, D. \& Fields, K. K. (1999). Borderline personality disorder and bone marrow transplantation: Ethical considerations and review. PsychoOncology, 8(1), 46-54.

Wettergren, L., Langius, A., Björkholm, M. \& Björvell, H. (1997). Physical and psychosocial functioning in patients undergoing autologus bone marrow transplantation: A prospective study. Bone Marrow Transplantation, 20, 497502.

Wingard, J. R. (1994). Functional ability and quality of life of patiens after allogeneic bone marrow transplantation. Bone Marrow Transplantation, 14(4),529-533.

Wolcott, D. L., Wellisch, D. K., Fawzy, F. I. \& Landsverk, J. (1986). Adaptation of adult bone marrow transplant recipient long-term survivors. Transplantation, 41(4), 478488.

Valles, M. S. (1997). Técnicas cualitativas de investigación social. Madrid: Síntesis.

Recebido em 27/05/2005 Aceito em 28/06/2006

Endereço para correspondência: Rodrigo Sanches Peres. Rua Jesuíno de Arruda, 2753,Centro, CEP 13560-060, São Carlos-SP. E-mail: rodrigosanchesperes@yahoo.com.br 